\title{
Seguridad y ciudades globales: Madrid, nuevo concepto de seguridad en el marco de los ODS
}

\author{
Pablo E. Rodríguez Pérez ${ }^{1}$
}

Recibido: 14/12/2020 / Aceptado: 08/07/2021

Resumen. El concepto de seguridad actualmente ha evolucionado hacia conceptos de protección individual de carácter humanístico, es decir, la seguridad adquiere dos elementos básicos. Por un lado, un concepto social de la seguridad, políticas dirigidas a un colectivo que podríamos relacionar con las políticas de seguridad ciudadana; y, por otro lado, un concepto individual de protección global de las personas frente a todos los elementos que puedan poner en peligro su desarrollo integral, lo que identificaríamos con la seguridad humana.

La evolución demográfica arroja datos cada vez más contundentes en la línea de que estos conceptos globales se dan en entornos urbanos con características de globalidad no pudiendo aplicar en estos casos políticas generalistas de carácter estatal o urbes con condicionantes y estructuras demográficas ambientales propias.

El modelo de seguridad global debe evolucionar y empezar a velar por la seguridad ciudadana y humana en entornos urbanos como modelo concreto de actuación. Esta seguridad urbana de naturaleza global viene a determinar un modelo de intervención de seguridad del siglo XXI, que intrínsecamente está relacionada con el modelo de los objetivos de desarrollo sostenible, muchos de ellos dirigidos a conseguir un entorno de libertad y de seguridad jurídica.

Por tanto, debemos encaminarnos a modelos de políticas públicas en seguridad global centralizadas en riesgos urbanos y de ejecución por las administraciones más cercanas al ciudadano.

Palabras clave: Políticas locales de seguridad, ciudades globales, riesgos globales, desarrollo sostenible.

\section{[en] Security and global cities: Madrid, a new concept of security in the framework of the SDGs}

Abstract. The concept of security has currently evolved towards concepts of individual protection of a humanistic nature, that is to say, security acquires two basic elements. On the one hand, a social concept of security, policies aimed at a group that we could relate to citizen security policies; and on the other hand, an individual concept of global protection of people against all elements that could endanger their integral development, which we would identify with human security.

Demographic evolution provides increasingly convincing evidence that these global concepts are found in urban environments with global characteristics, and in these cases it is not possible to apply generalist policies of a state nature or cities with their own environmental demographic conditions and structures.

The global security model must evolve and begin to look after citizen and human security in urban environments as a specific model of action. This global urban security determines a 21 st century security intervention model, which is intrinsically related to the model of sustainable development objectives, many of which are aimed at achieving an environment of freedom and legal security.

Therefore, we must move towards models of public policies on global security centralised on urban risks and implemented by the administrations closest to the citizen.

Keywords: Local security policies, global cities, global risks, sustainable development.

Sumario. 1. Introducción. 2. Las ciudades globales. 3. Madrid: ciudad global. 4. Los nuevos retos para las políticas de seguridad en Madrid. 5. Un enfoque global. La aplicabilidad de los “ODS” en la seguridad pública madrileña. Conclusiones. Bibliografía.

Cómo citar: Rodríguez Pérez, P. E. (2021): Seguridad y ciudades globales: Madrid, nuevo concepto de seguridad en el marco de los ODS, en Cuadernos de Gobierno y Administración Pública 8-1, 105-118.

\footnotetext{
Director General de Policía Municipal del Ayuntamiento de Madrid rodriguezppe@madrid.es
} 


\section{Introducción}

La crisis sanitaria que actualmente estamos padeciendo ha traído consigo un cambio brusco y en algunos casos de gran calado en el comportamiento de todos los habitantes de nuestro planeta desde las localidades más pequeñas a las urbes globales y es en estas últimas donde más se marcan estas nuevas estructuras de comportamiento social, debido al número elevado de población en las mismas.

La crisis sanitaria del COVID 19 ha puesto el acento en comportamiento y en políticas que tienen un destacado signo en la temporalidad. No podemos decidir a grandes o medios plazos, al revés, debemos de tomar medidas cortoplacistas, que vayan derivándose del comportamiento de la enfermedad en nuestros ciudadanos y ciudadanas, y en indicadores como las acumulaciones de casos de infección, el número de recursos hospitalarios, los índices de concentración de casos y ratios por población. En una palabra se crea un nuevo marco de planificación que es la objetivación de la políticas en general de las instituciones públicas, sea cual sea la competente, el Estado, las Regiones o Comunidades Autónomas y obviamente las entidades locales, que son las que de manera inmediata tienen que dar soluciones a la población y a la administración que demanda estas soluciones, con la circunstancia añadida que son de mucho calado e importancia para la vida ya no social, sino íntima y de supervivencia de parte de la sociedad a la que sirven.

Esta pandemia, que desde el pasado año está asolando al mundo y que tiene un reflejo en lo más particular de nuestro comportamiento, es decir, pasa de lo universal a lo inmediatamente individual, tiene su reflejo en las consecuencias de la lucha contra la misma, ya que una pandemia de origen vírico y de solución científica, tiene a corto y medio plazo una solución en el plano de la seguridad.

Si observamos la lucha que con carácter general hasta el día de hoy han dado todos los países para tratar de prevenir y paliar los efectos del COVID 19, todos tienen un denominador común: restringir el movimiento de las personas. Desde China donde a finales del año 2019 empezaron a saltar las alarmas sobre esta enfermedad desconocida desde el punto de vista sintomatológico y epidemiológico y de su curación hasta el día de hoy, de una manera u otra y en tanto en cuanto el mundo científico trata de buscar un solución farmacológica a la misma, las medidas de prevención de la enfermedad y paliación de efectos y obviamente de impedir la propagación de la misma ha sido la de restringir los movimientos de las personas para que el virus tampoco se desplace, antes o después, en la llamada primera ola o en la que actualmente se describe como segunda ola, las administraciones restringen los derechos individuales de los ciudadanos a través de diferentes medidas que de manera coloquial y a mi juicio poco acertada se han venido describiendo como Confinamientos, Toques de queda, etc., que tienen una raíz mucho más restrictiva y de claro talante belicista muy apegado al primera mitad del Siglo XX.
Teniendo en cuenta esta restricción de movimientos individuales y sociales como denominador común de las políticas institucionales, las mismas han pasado también por una evolución significativa ya que de una restricción de movimientos muy concreta en los domicilios y que tenía contadas excepciones, en la actualidad se restringen actividades sociales para tratar de conseguir, de manera indirecta, este control de movimientos sociales y por tanto de cada ciudadano, pero también preservando en la medida de lo posible el equilibrio con los bienes productivos y el trabajo.

La derivada primera de este tipo de políticas sanitarias, enfocadas en comportamientos sociales y su control, lleva directamente a políticas de seguridad que son la plasmación activa de las medidas que la administraciones públicas deben hacer efectivas y eficaces, de ahí como señalé anteriormente deriva el silogismo de que un problema de orden sanitario tiene un reflejo de marcado carácter de seguridad y orden público que en estos momentos se vuelve más concreto y marcado.

Pero señalaba esta derivada directa e importante de la seguridad, como consecuencia de las medidas de prevención y paliación de los efectos de la enfermedad, pero hay otros de calado vital en la sociedad como son los de carácter económico, especialmente en sector servicios y nuestro país, es un claro ejemplo de economía basada principalmente en los servicios. La limitación de movimientos, las restricciones colectivas e individuales traen consigo directamente una bajada de la demanda de este tipo de productos, lo que lleva aparejado que empresas de sectores directamente e indirectamente asociados al consumo social se vean directamente afectadas y deban de parar de manera drástica su actividad productiva y empresarial, lo que trae consigo un reducción de las ofertas de trabajo y un número elevado de personas que pierden sus empleos y salvo ayudas de carácter estructural o coyuntural de las administraciones públicas no tiene recursos para su vida diaria, lo que también se refleja en un problema de seguridad en este caso más global y que podemos enmarcar en la estricta respuesta de la protección civil como institución de dar cobertura y reparación a las consecuencias de una gran catástrofe o calamidad pública.

Es obvio que lo urgente se vuelve emergente en estos tiempos de pandemia universal, pero frente a esta emergencia también la vida sigue, y por tanto el desarrollo de la planificación de políticas generales deben cohabitar con las políticas de emergencia y es un duro y difícil equilibrio para las políticas públicas y especialmente para las políticas de seguridad, ya que se yuxtaponen las soluciones de control de medidas de seguridad sanitaria con las propias de la seguridad ciudadana y administrativa que se siguen dando, si cabe en algunos casos enmascaradas con el objetivo cortoplacista de las medidas sanitarias. Hay que seguir controlando los delitos contra la propiedad, las ocupaciones de vivienda y usurpaciones, la propiedad intelectual, los delitos contra la salud pública y las medidas contra la siniestralidad laboral y vial, como elementos estructurales de la gestión de la vida de los ciudadanos, más aún en las grandes ciudades. 


\section{Las ciudades globales}

Según algunos de los estudios demográficos más recientes, en el año 2050, la población de las ciudades estará en torno al $68 \%$ de la población mundial, absorbiendo en este nuevo espectro sociológico las ciudades los elementos típicos de los estados actuales, lo que algunos autores han descrito como la vuelta a las Ciudades-Estado de la Edad Media, pero claro está con un avance tecnológico nunca conocido hasta este momento.

Esta globalización tiene un elemento eminentemente tecnológico y será la actividad en este campo lo que permita un desarrollo más potente en los próximos años a las ciudades, especialmente con el diseño de las llamadas smart-cities. Pero no debemos de pasar por alto que este nuevo empoderamiento de las ciudades desde el punto de vista tecnológico, necesita las instituciones que nos gobiernan, del factor ciudadano, el ser humano por encima de la máquina o de la tecnología asociada a la misma. El centro de las políticas públicas ha sido y debe ser en este siglo el cuidado global de los habitantes de cada una de ellas con las particularidades que presentan cada una de ellas dependiendo de la sociedad en la que están enmarcadas.

En la actualidad la existencia de metrópolis globales trae consigo que varios autores empiecen a considerar que las ciudades globales ( para reforzar el sentido de la "glocalidad" en los riesgos mundiales), tiene un tejido de infraestructuras y digitalización que las hace estar por encima de la propia noción de "comunidad nacional"; definiéndose las mismas como una nueva zona fronteriza entre las urbes tradicionalmente entendidas y los Estados que a juicio de la Profesora Sassen " se reconfigura como un espacio parcialmente desnacionalizado que permite las políticas subnacionales como las trasnacionales. Lo político se implanta y se reinventa a partir de un amplio espectro de intereses...Parte de lo político en cada ciudad empieza a orientarse hacia una red interurbana que va más allá de cada ciudad (multi-sited-network) ..., las ciudades se han convertido en un terreno estratégico para un conjunto importante de conflictos y contradicciones...las ciudades globales es una vez más la escala donde se materializa las dinámicas estratégicas, tanto económicas como políticas" (Sassen, 2017).

Uno de los elementos a tener cuenta dentro de estas ciudades globales en el campo de la seguridad es el factor humano y su problemática global. No se pueden aplicar parámetros estatales únicamente en dar respuesta a la seguridad como concepto antiguo de orden público o meramente de seguridad ciudadana en la protección de los derechos y libertades públicas, sino que ésta debe de tener un horizonte más global desde el punto de vista individual y tener su epicentro en la persona y darles una respuesta global a sus necesidades de seguridad; se debe ir que la ciudad pase a ser el centro de esta respuesta de manera más inmediata.

Actualmente las políticas de seguridad se marcan desde los Estados y es desde éstos desde los que se articulan las instituciones primarias en dar una respuesta adecuada a las necesidades de los habitantes o población de las ciudades, con mayor intensidad en las grandes ciudades, pero considero que los Estados deben trasladar su competencia ejecutiva de manera efectiva en los próximos años, centrándose las políticas de seguridad globales en las ciudades globales y la coordinación de información y análisis de parámetros de seguridad en los Estados.

Si nos fijamos en el ámbito de la seguridad española, a mi juicio está anclada en situaciones sociológicas muy diferentes a las actuales. La ley de 1986 da respuesta a condiciones políticas y sociológicas muy diferentes a las actuales, muy centralizada y con claros síntomas de agradar a comunidades autónomas muy concretas con un desarrollo global que ahora no se da. No podemos comparar las sociedades urbanas del año 1986 con las sociedades urbanas y tecnológicas del año 2020. Podríamos señalar que la seguridad que compromete a la ley de 1986 es una seguridad de orden público de carácter centralizado con visos autonómicos y la seguridad actual es municipal con tintes autonómicos. Conceptos de ciudades globales, smart-cities, avances tecnológicos (5G) y globalización 4.0 marcan una distancia abismal con una sociedad tecnológica empezando a desarrollarse donde las interconexiones entre las personas eran de una tecnológica incipiente (telefónica y bolígrafo si se me permite).

Los modelos de la seguridad global deben cambiar, y no son novedosas las ideas que en la actualidad deben empezar a diseñarse de manera efectiva, y que las leyes deben ser un reflejo de la sociedad y no de meras circunstancias interesadas de las agendas políticas o de los operadores. Debemos de empezar a hablar de la seguridad de las ciudades globales de manera directa, donde a mi juicio los regidores de estas ciudades tengan un peso específico en los diseños de seguridad y donde los operadores de seguridad locales sean tenidos en cuenta de manera efectiva.

Cuando se habla a título de ejemplo de la "España Vaciada", es porque de algún modo u otro existe una "España Llenada" (permítaseme la antinomia semántica), y esta no se da en otros lugares que en las ciudades o en sus coronas metropolitanas, donde la tendencia es a subir paulatinamente de población, lo que conlleva directamente a reconocer la importancia que en unos años este sector de la seguridad urbana representará en las políticas públicas de las administraciones competentes en ellas y el giro, a mi parecer cada vez más necesario, que se debe dar a la distribución competencial en materia de seguridad.

Si aplicáramos los términos que la teoría del riesgo del siglo pasado, que tan acertadamente pronosticó un cambio en las ideas de la seguridad con el concepto "glocalidad", este término adquiere ahora un nuevo sentido al poner más el acento en lo local; ya que cada vez en más ocasiones se debe de dar una respuesta a los ciudadanos desde el ámbito de la administración local más cercano al ciudadano.

Desde esta perspectiva es necesario el cambio de las normas jurídicas en materia de seguridad tanto desde la perspectiva pública como privada (más de 5 años llevamos esperando un reglamento de seguridad privada). Este cambio normativo debe de poner el acento en 
Gráfico 1. Evolución del número de habitantes en el municipio de Madrid.

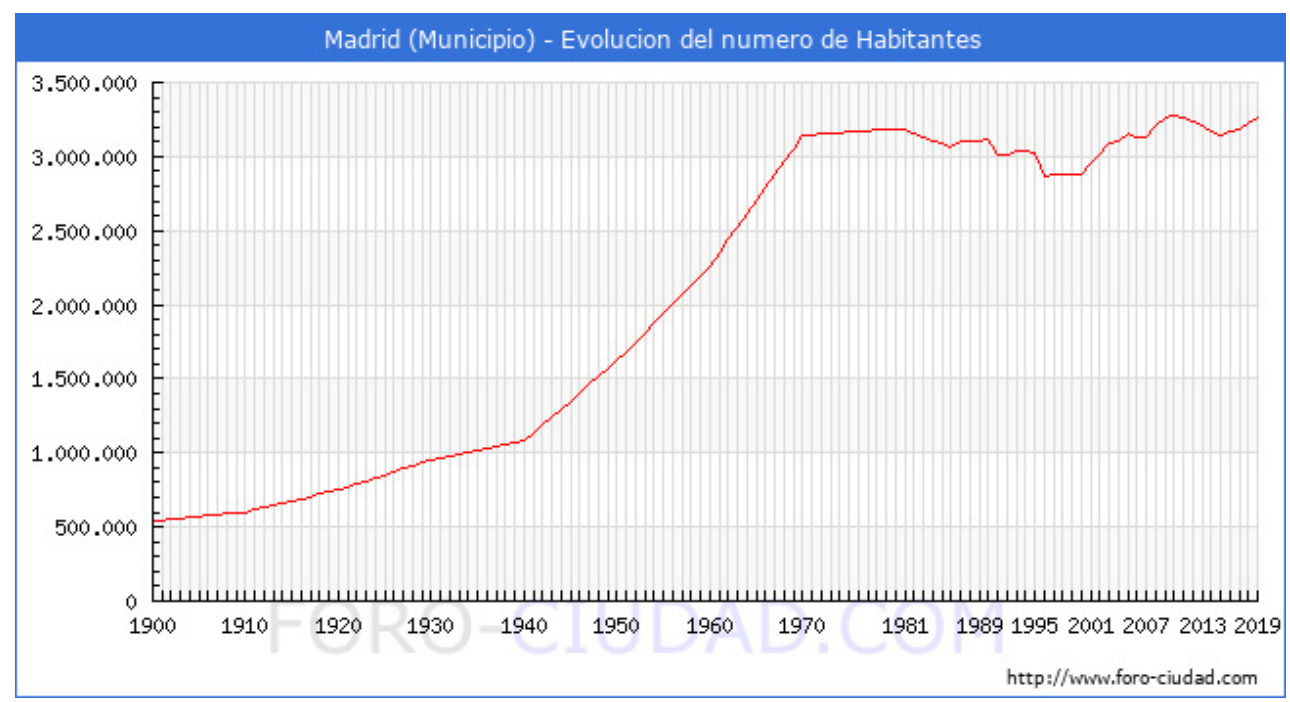

Fuente: www.foro-ciudad.com.

las ciudades globales como elemento determinante de ejecución de políticas de seguridad y alcanzando los alcaldes la competencia en esta ejecución a través de sus propias instituciones de manera decidida. Hemos podido observar y observamos en la actualidad con la crisis sanitaria del COVID 19, cómo son los regidores los máximos responsables de dar solución global a las necesidades de los vecinos, de sus necesidades humanas, lo que nos liga directamente con el concepto de seguridad humana por encima de la seguridad ciudadana y del orden público, más propio de normativas estatales centralistas que no son conocedoras de las diferencias de las sociedades y del conocimiento de la urdimbre social para las que actúan.

El Estado debe tener la capacidad de coordinación y de información de los objetivos generales de las políticas de seguridad siendo las ciudades globales y en su defecto las entidades provinciales en su caso las que ejecuten de manera decidida esas políticas de seguridad, teniendo en cuenta cada vez más las interconexiones internacionales de estados con estados (Unión Europea) y ciudades con ciudades globales, con conexiones políticas necesarias para dar efectividad a su propias políticas económicas y de servicios.

Teniendo en cuenta los criterios asociados a las grandes poblaciones a través una nueva realidad social, las soluciones a los problemas de los ciudadanos que en ellas residen son demandados a la administración más cercana y con interconexiones a diferentes servicios, y esta cercanía solamente la da la administración local. La solución a diferentes vectores de problemas que están, de una forma u otra ligados al ámbito del seguridad humana a través de la seguridad sanitaria, ciudadana, medioambiental, económica y de estado de bienestar.

La seguridad humana se constituye como un nuevo elemento de las grandes ciudades y factor. Las políticas públicas de seguridad y protección civil vienen a determinar como base de su concepto la interconexión a la respuesta de factores no solo de orden público sino centrado en la Seguridad.
Este nuevo concepto acuñado a partir de la Cumbre del Milenio de Seguridad, realizado bajo el amparo de la ONU en 2005(resolución 60/1 de la Asamblea General), y que posteriormente las conclusiones de la misma y de otras sesiones especiales en materia de seguridad humana, llevaron a la firma por parte del Secretario General en el 2010 del informe sobre seguridad humana en el que se pone de relieve toda la problemática actual desde el plano de la seguridad del Siglo XXI. Son múltiples definiciones las que se han establecido sobre seguridad humana, internacionales y en comités regionales como "proteger el núcleo vital de toda vida humana de manera que mejore las libertades básicas y las relaciones humanas" o "proteger el núcleo vital de todas las vidas humanas de manera que mejoren las libertades humanas"2. De esta manera y siguiendo el desarrollo del texto del Secretario General de Naciones Unidad, la seguridad humana tiene a través de sus diferentes definiciones tres conceptos esenciales:

a) La seguridad humana es una respuesta a los problemas actuales y emergentes.

b) La seguridad humana exige una expansión-expansión de la comprensión de la seguridad, donde se forma la protección y el empoderamiento de las personas que son las bases y el progreso de la sociedad.

c) La seguridad humana no implica el uso de la fuerza $\mathrm{y}$ tiene como objeto integrar a todos los ciudadanos.

Así mismo los principios en lo que reside la seguridad humana son: ${ }^{3}$

a) Centrada en las personas. Para la seguridad humana las personas son el centro del análisis $y$,

\footnotetext{
Unit Nations. General Assembly. Human Security. Report of the Secretary General. Sixty-fourth Sesion. 8 March 2010. A/64/701

Unit Nations. General Assembly. Human Security. Report of the Secretary General. Pág. 7
} 
consecuentemente, se consideran las condiciones que amenazan la sobrevivencia, medios de vida y dignidad de las personas.

b) Multisectorial. La seguridad humana se basa en la comprensión multisectorial de las inseguridades. En consecuencia, además de la seguridad nacional, la seguridad humana implica la comprensión de una gama amplia de amenazas y de sus diferentes posibles causas relacionadas con la economía, la alimentación, la salud, el medio ambiente, la seguridad personal, comunitaria y política.

c) Integral. La seguridad humana implica enfoques integrales que enfatizan en la necesidad de respuestas comprensivas y multisectoriales con el fin de articular las agendas que se relacionan con seguridad, desarrollo y derechos humanos.

d) Contextualizada. La seguridad humana reconoce que las inseguridades varían considerablemente en diferentes contextos y, por lo tanto, promueve la búsqueda de soluciones contextualizadas que respondan adecuadamente a cada situación particular.

e) Preventiva. Al llegar a las causas y a las manifestaciones de las inseguridades, la seguridad humana se orienta a la prevención e introduce sus estrategias de protección y empoderamiento.

Por lo tanto debemos partir de tres conceptos que actualmente se interrelacionan principalmente en el ámbito de las ciudades globales: Las ciudades son el nuevo paradigma del siglo XXI como modelos a escala del comportamiento social de los países y por tanto deben tener políticas públicas asociadas a sus propias características; las amenazas a la sociedad y especialmente en las ciudades de características globales tienen interconexión entre ellas o derivan las unas de las otras, así la actual pandemia derivada del COVID 19 ha traído consigo una crisis económica y a su vez una crisis alimenticia $^{4}$ en algunos sectores de la población; y tercero y más importante, las políticas institucionales de los gobiernos locales deben dar una respuesta a todas estas amenazas que se presentan como primer respondiente político y social y por tanto esta respuesta global, nos lleva a un concepto de seguridad hasta ahora altamente descrito pero no concretado que es el de seguridad de respuesta global se ha definido como seguridad humana.

Desde un plano más "regional", también la Unión Europea está en la actualidad marcando las líneas de la estrategia para la "Unión de la Seguridad"5señalando en su preámbulo que la "seguridad no es solo el fundamento de la seguridad personal, sino que también protege los derechos fundamentales y sienta la base para la confian-

\footnotetext{
El Reino Unido es la quinta economía del mundo, con un PIB per cápita cuatro veces superior al promedio mundial. Sin embargo, las cifras de UNICEF muestran que el 19\% de los niños menores de 15 años en el Reino Unido viven con adultos que luchan por comprar alimentos, entre las peores tasas de Europa, pero a la par con los Estados Unidos. Los niños tienen tanta hambre en una ciudad británica que están comiendo de los contenedores. Tiempos desesperados, medidas desesperadas. Simon Dawson.07 de febrero de 2019. World Economics Forum Events

5 Estrategia de la Unión de la Seguridad de la UE 2020 - 2025. Bruselas 24 de julio de 2020. COM (2020)
}

za y el dinamismo en nuestra economía, nuestra sociedad y nuestra democracia", lo que nos recuerda de igual manera un concepto más amplio de la seguridad ciudadana propiamente dicha y se centra en una seguridad de carácter extensivo y global en todo lo que representa el ciudadano y el entorno social que le rodea y su relación con el mismo. Señala también que "la globalización, la libre circulación y la transformación digital siguen aportando prosperidad, facilitando nuestras vidas e impulsando la innovación y el crecimiento. Pero estos beneficios llevan aparejados riesgo y costes inherentes". Hace referencia constante la estrategia europea, como no puede ser de otra manera y como lo hemos hecho en nuestro análisis de seguridad en ciudades globales, a la crisis del COVID 19, que ha representado un cambio de paradigma en los conceptos básicos que tuvimos de seguridad y respuesta a las amenazas; y que éstas han creado nuevas oportunidades de delincuencia cuyas consecuencias podrían haber puesto en peligro el sistema institucional de respuesta por parte de las administraciones comprometidas en la paliación y prevención de esta pandemia, especialmente en un contexto de divisiones sociales y vulnerabilidad en ciertos sectores de la población en materia de seguridad humana.

Señalar que el sistema europeo de seguridad y su estrategia para 2020 a 2025 parte de los siguientes objetivos para su desarrollo:

a) Desarrollar capacidades y medios para la detección temprana, la prevención y la respuesta rápida a las crisis

b) Centrarse en los resultados.

c) Conectar a todos los actores de los sectores públicos y privados en un esfuerzo común

Estos objetivos se realizan a través de una serie de prioridades funcionales que tienen como base argumental la libertad y los derechos fundamentales de los ciudadanos: Un entorno de seguridad con garantías de futuro, hacer frente a las amenazas cambiantes, proteger a los europeos del terrorismo y la delincuencia organizada y por ultimo un ecosistema europeo de seguridad sólido. ${ }^{6}$ La seguridad humana impregna toda la estrategia de la Unión Europea, pero a mi juicio adolece de valentía suficiente, para hacer una referencia de seguridad propia de las ciudades globales europeas, teniendo en cuenta que en el continente europeo se encuentra más de 10 de las 20 ciudades globales del mundo, particularmente dos de las tres primeras ciudades globales como son Londres y Paris. Considero que la seguridad estratégica de la Unión Europea especialmente tiene que tener una referencia explícita a las grandes áreas metropolitanas lideradas por las ciudades globales. Es obvio que el carácter nacional debe ser el entorno estratégico fundamental de la Unión Europea, pero debemos señalar que este tipo de urbes tienen entre sí una urdimbre social que va más allá de la propia seguridad nacional y les hace merecedoras de una estrategia derivada, si no propia.

Estrategia de la Unión de la Seguridad de la UE 2020 - 2025. Bruselas 24 de julio de 2020. COM (2020) 
Tal como se ha visto recientemente, los atentados terroristas se producen en las ciudades globales europeas o en vías de serlo, Madrid, Londres, París, Viena, Berlín y Barcelona entre ellas. Además, las grandes compañías "delictivas" por su propia naturaleza se ubican en centros empresariales con grandes servicios de comunicaciones y transferencia de comunicaciones, para la planificación de grupos u organismos dedicados a la delincuencia, blanqueando los productos de sus hechos delictivos.

En términos generales el análisis Lloyd's analiza en las ciudades globales tendencias importantes:

a) Las economías emergentes cargaran con una proporción mayor de las pérdidas financieras relacionadas con el riesgo como resultado de su crecimiento económico.

b) Las amenazas causadas por el hombre cada vez tienen más relevancia, entre las que destacan los desplomes bursátiles, ciberataques, accidentes nucleares. Estos significan un cuarto de las pérdidas potenciales de todas las ciudades.

c) El impacto de los riesgos nuevos o emergentes (ciberataques, pandemias) es cada vez mayor.

d) La población en las ciudades cada vez es mayor, se está desplazando de las zonas rurales.

e) Las amenazas son algo cambiante pero cada vez los causados por el hombre está ganando en la medida en que las empresas se están expandiendo a nuevos mercados y territorios.

f) La cifra total en la que se calculan los riesgos (amenazas y tendencias) se cifra en el estudio en 4,56 billones de dólares, siendo el concepto económico del riesgo (coincide el estudio de ciudades con el de Davos) y en concreto el desplome de las economías y mercados bursátiles el más importante con un coste de 1,05 billones de dólares. Las amenazas que representan el 91\% de la cuantificación de costes de los riesgos, podemos clasificarlas (siguiendo la clasificación de Davos) de la siguiente manera medioambientales 4 , sociales (seguridad) 2, económicos 2 y tecnológicas 1 . Se concretan en los siguientes ejemplos: sequias, inundaciones, terremotos, vendavales, terrorismo, pandemias, ciberataques, impagos de la deuda, crisis de los precios del petróleo, desplome de las economías emergentes ${ }^{7}$.

\section{Madrid: ciudad global}

Madrid es una ciudad global, teniendo en cuenta los criterios que hemos apuntado en los epígrafes anteriores, $\mathrm{y}$ que podemos señalar como características: su territorio, la población, que es líder en las actividades económicas

Índices de Riesgo de las Ciudades.2015-2025. Resumen Ejecutivo. Pág. 1 Realizado en cooperación con la Universidad de Cambridge y LLoyds Bussiness School. https://www.lloyds.com/ /media/files/ news $\% 20$ and $\% 20$ insight $/$ risk $\% 20$ insight $/ 2015 /$ city $\% 20$ risk $\% 20$ index/city $\% 20$ risk $\% 20$ index $\% 20$ executive $\% 20$ summary_spanish.pdf. nacionales e internacionales y es imagen de los servicios globales del país ${ }^{8}$.

Son muchos los diferentes estudios sociológicos y estadísticos para cuantificar la posición y calidad de las ciudades en el mundo y la importancia de cada una de ellas. Así por ejemplo, el realizado por el Instituto de Estrategias Urbanas de la Fundación japonesa MORI publica el ranking Global Power Cities Índex $1019^{9}$ señala que Madrid se encuentra en el puesto 13 de las ciudades más poderosas del mundo, ostentando el 5 lugar de las ciudades europeas, solo por detrás de Londres, Paris, Ámsterdam y Berlín, midiendo aspectos tan destacados como las actividades de negocio, el capital humano, el intercambio de información y telecomunicaciones, las actividades culturales y el compromiso político con la ciudad.

Otro estudio destacado sobre ciudades globales es el realizado por el Grupo de Estudios sobre Globalización y Ciudades Mundiales más conocido por sus siglas $\mathrm{GAWC}^{10}$, que clasifica igualmente en tres categorías las ciudades Alfa, Beta y Gamma, situando en el grupo Alfa a Madrid y también en la posición 5 dentro del ámbito europeo.

Por parte del ayuntamiento de Madrid, se publicó en diciembre del año 2019, el posicionamiento global de Madrid en el mundo ${ }^{11}$ con referencia a los criterios de globalidad que hemos estado analizando en este análisis de la seguridad en ciudades globales, valorando una serie de criterios como el del posicionamiento en el entorno internacional, la calidad de vida, la accesibilidad, el talento, los negocios y el poder de atracción de Madrid en este campo la innovación como consecuencia de este nuevo reto para la ciudad y su sostenibilidad y sus interconexiones.

En cuanto a las ciudades más poderosas del mundo Madrid es la $13^{\mathrm{a}}$ ciudad global con mayor poder para atraer personas, capitales y empresas de todo el mundo y la $5^{\mathrm{a}}$ de Europa. Es importante señalar el aumento exponencial que Madrid ha conseguido en lo referente a la habitabilidad donde escala a la tercera posición mundial desde el año pasado y donde se miden factores especialmente vinculados con el concepto de seguridad humana, es decir el bienestar de los ciudadanos que viven en ella,

\footnotetext{
WEF. Muggan, Robert. Las ciudades más frágiles. "La expansión de las ciudades están reconfigurando la política global y económica.... Hoy en día, las cadenas de suministros globales pasan por ellas... creándose nuevas formas innovadoras de cooperación internacional, uniéndose para exigir compromisos más estrictos en materia de cambio climático, apoyo a los grupos de emigrantes y recursos para combatir el extremismo. Hoy en día hay aproximadamente 200 redes interurbanas, más que el número de asociaciones interestatales." Fecha del informe 7/02/2016.

9 En: https://mori-m-foundation.or.jp/english/ius2/gpci2/2019.shtml GPDI 2019. Hace referencia a que Madrid ha subido en 5 años del puesto 17 al puesto 13 debido al aumento de su PIB y al control de la congestión del tráfico. Pág. 2

10 La ontología de la ciudad-región global: una crítica de la estadidad .P. Perulli Las regiones urbanas mundiales de Europa participan constantemente en las estrategias europeas. Sin embargo, su papel en la formulación de políticas europeas es limitado, si no irrelevante. Esta aparente paradoja se explicará por el papel interesado de los Estados-Nación para ser considerados los órganos representativos de "sus" regiones urbanas mundiales. www.lboro.ac.uk/gawc/rb/rb415. 11 Madrid en el Mundo. Informe del Posicionamiento Global 2020. Ayuntamiento de Madrid. Área de Gobierno de Economía, Innovación y Empleo. Diciembre 2019
} 
la esperanza de vida, el coste de la vida, el entorno laboral y principalmente la seguridad objetiva y subjetiva. En este concepto de seguridad Madrid también mejora posiciones ( 5 en concreto) como ciudad global con mayor proyección de futuro.

En referencia al bienestar de los ciudadanos como factor intrínseco de la seguridad humana teniendo en cuenta los factores de estrategia de seguridad global y la resiliencia de las ciudades globales en cuanto a las diferentes amenazas y riesgos y su modo de afrontarlos, Madrid como ciudad global destaca principalmente en este capítulo de estudio. Madrid se sitúa como la primera ciudad con mayor esperanza de vida de toda Europa, según el sondeo estadístico realizado por la agencia Eurostat ( $1^{\mathrm{a}}$ de 367 ciudades $)^{12}$ siendo la esperanza de vida de 84,9 años, lo que nos da un elemento muy importante a la hora de establecer estrategias de seguridad humana trasversales por la diferencia de criterios subjetivos que tienen las franjas de edad a las que van dirigidas las políticas de prevención y el acceso a las redes de información actuales ( por ejemplo internet y los ciberdelitos en estos sectores vulnerables).

Madrid es una ciudad segura, a tenor de lo que refieren el estudio ya señalado del Ayuntamiento de Madrid y los índices de referencia que se manejan en él. The Economist, publica el "Safe Cities Index"13 que analiza en nivel de seguridad de 60 ciudades del mundo. Dicho estudio analiza: la seguridad digital, la seguridad de la salud, la seguridad en las infraestructuras y la seguridad personal; destacando sobremanera en la seguridad de las infraestructuras ocupando el $5^{\circ}$ puesto mundial y en cuanto a la seguridad personas ocupa el puesto $18^{\circ}$ (en cuanto a la seguridad sanitaria se encuentra en el puesto 20 y el peor resultado en el año 2019 lo sitúa en el puesto 28 de la seguridad digital), siempre por encima de la media mundial. El referido estudio indica que la "gestión urbana jugará un papel fundamental en la definición de la calidad de vida de la mayoría de los seres humanos en los próximos años. Un elemento clave de esto será la capacidad de las ciudades para brindar seguridad a sus residentes, negocios y visitantes."

Como apunte anteriormente, el Informe The Economics también asevera que la "resiliencia urbana" es un factor fundamental de futuro. El informe matiza que "la capacidad de la ciudades para absorber y recuperarse de los choques, ha tenido una influencia cada vez mayor en el pensamiento sobre la seguridad urbana durante la última década, especialmente porque lo legisladores se preocupan por las implicaciones del cambio climático". Por este motivo el informe también evalúa las políticas desarrolladas para el riesgo en desastres.

Este posicionamiento nos da una idea de la situación de Madrid en el mundo y especialmente de las referencias con otras ciudades, permitiéndonos señalar que es muy positiva, si bien existen áreas de mejora que deben de orientar los objetivos de seguridad dentro de las polí-

12 Madrid en el Mundo. Informe del Posicionamiento Global 2020. Ayuntamiento de Madrid. Área de Gobierno de Economía, Innovación y Empleo. Diciembre 2019. Pág. 22

13 En: https://safecities.economist.com/safe-cities-index-2019/. ticas globales de transversalidad que se deben de llevar en nuestra capital.

\section{Los nuevos retos para las políticas de seguridad en Madrid. Un enfoque global}

En la actualidad estas áreas de mejora de carácter estructural se contemplan en el marco de la Agenda 2030 ${ }^{14}$, que tiene su origen en el Convenio de Paris de Naciones Unidas sobre el cambio climático de 12 de Diciembre de $2015^{15}$; en la que se marca como compromiso que se informe de las contribuciones de las partes cada 5 años hasta el horizonte temporal de 2030.

En esta nueva agenda urbana se sostiene que las ciudades son el elemento esencial en el cumplimiento de los protocolos contra el cambio climático y por lo tanto se alinean expresamente con las políticas generales, como así se establece en el objetivo 11 del Tratado de Paris; que se deben de llevar a cabo en los países y especialmente en las grandes ciudades; es importante señalar que la Nueva Agenda Urbana ${ }^{16}$ en su preámbulo indica que "... la Nueva Agenda Urbana presenta un cambio de paradigma basado en la ciencia de las ciudades; establece normas y principios de planificación, construcción, desarrollo, gestión y mejora de las zonas urbanas en sus cinco pilares de aplicación principales: políticas urbanas nacionales, legislación y normativas urbanas, planificación y diseño urbano, economía local y finanzas municipales e implementación local"'17.

Dentro del programa de Desarrollo Urbano sostenible, los compromisarios se comprometen entre otras cuestiones a:

14 Nueva Agenda Urbana en la Conferencia de Naciones Unidad y el Desarrollo Urbano Sostenible (Hábitat III) celebrada en Quito del 17 al 20 de octubre de 2016 que tuvo el refrendo de la Asamblea General de Naciones Unida el 23 de diciembre de 2016.

15 Conferencia de la Partes Paris de 30 de noviembre a 11 de diciembre de 2015. Plataforma de Durban para una acción reforzada.

16 Resolución aprobada por la Asamblea General el 25 de septiembre de 2015. 70/1. Transformar nuestro mundo: la Agenda 2030 para el Desarrollo Sostenible. Septuagésimo período de sesiones Temas 15 y 116 del programa. Asamblea General.

17 Antonio Sampario. Reseach associeate for security and development, international institute for strategic studies. 10/11/2016 weforum.org. Señala un cambio significativo respecto a los documentos anteriores, ya que vincula directamente el desarrollo urbano con la necesidad de prestar "atención especial" a los países que se encuentran en transiciones posteriores a conflictos. Aún más prometedora es la promesa de "integrar medidas inclusivas para la seguridad urbana, la prevención de la delincuencia y la violencia", incluido el terrorismo, "en el desarrollo de estrategias e iniciativas urbanas".

El mismo razonamiento - que un enfoque local puede marcar la diferencia en la respuesta a los conflictos armados - subrayó el lanzamiento, a principios de este año, de la Alianza Global para Crisis Urbanas, una asociación entre 65 organizaciones que desean planear, prevenir y responder mejor a los conflictos urbanos Y el desplazamiento. Asimismo, el nexo entre las tendencias urbanísticas y los conflictos ha llevado al Comité Internacional de la Cruz Roja a involucrarse intensamente en las negociaciones para la Nueva Agenda Urbana, llamando públicamente a que los líderes y pensadores urbanos "recuerden a los millones de personas que viven en zonas urbanas violencia".

Ahora imagine si un nuevo flujo de pensadores de estrategia y seguridad puede sacar provecho del desarrollo urbano, la planificación y las tecnologías para hacer más difícil que los grupos armados no estatales operen en las ciudades. Este es el potencial de la Nueva Agenda Urbana.

Actuar localmente para cambiar a escala global 
“ii) El fortalecimiento de la gobernanza urbana, con instituciones sólidas y mecanismos que empoderen e incluyan a los interesados de las zonas urbanas, así como los mecanismos de control adecuados, que faciliten una mayor previsibilidad y coherencia en los planes de desarrollo urbano para promover la inclusión social, un crecimiento económico sostenido, inclusivo y sostenible y la protección del medio ambiente".

En este sentido se estructuran por las partes una serie de compromisos todos ligados al concepto de seguridad humana, si bien en el criterio 30 señala concretamente en cuanto a la efectiva seguridad conceptual de los habitantes de las ciudades lo siguiente : "Nos comprometemos a promover un entorno seguro, saludable e inclusivo en las ciudades y los asentamientos humanos que permitan todos vivir, trabajar y participar en la vida urbana sin temor a la violencia y la intimidación, teniendo en cuenta que las mujeres y las niñas, los niños y los jóvenes y las personas en situación vulnerable suelen verse especialmente afectados. Trabajaremos también en pro de la eliminación de las prácticas nocivas contra las mujeres y niñas, como el matrimonio infantil, precoz y forzado y la mutilación genital femenina".

Regionalmente Eurostat, ha considerado 100 indicadores a nivel de Objetivos de Desarrollo Sostenibles (en adelante ODS).

Consecuentemente al Tratado de París y en el ámbito urbano Hábitat como reflejo del anterior, por parte del Gobierno Español en el año $2018^{18}$, publica en referencia a los indicadores de seguimiento de Naciones Unidas del 2017, un sistema de seguimiento que tiene como marco los 232 indicadores globales de seguimiento que según sus propias palabras "están concebidos para permitir el seguimiento del progreso a escala mundial, al tiempo que permite analizar la situación comparada y sirve de estímulo al progreso de cada país".

En referencia a este proceso de implementación y especialmente con respecto a las entidades locales el Gobierno Español, el 18 de julio de 2018, estableció el Plan de Acción para la implementación de la agenda 2030 ("Hacia una Estrategia Española de Desarrollo Sostenible", en cuyo epígrafe 5, señala el compromiso de las entidades locales) ${ }^{19}$.

Resaltar que en estos compromisos se hace referencia a un nuevo sentido del término como "localización"20 cuando señala que "...Estos objetivos no pueden alcanzarse sin tener en cuenta la necesidad de fortalecer las capacidades de los gobiernos locales incluyendo el desarrollo de herramientas participativas, innovadoras y sostenibles que garanticen la apropiación por parte de la ciudadanía". Es fundamental la categorización de este nuevo empoderamiento de las ciudades cara a un futuro en el que soportaran el $68 \%$ de la población del planeta, es imperativo, y deberíamos pensar que sería un error fatal que el empoderamiento de las ciudades

\footnotetext{
18 En: www.agenda2030.gob.es

19 Plan de Acción para la Implementación de la Agenda2030. Hacia una Estrategia Española de Desarrollo Sostenible. En: www.agenda2030. gob.es

20 Plan de Acción para la Implementación de la Agenda2030. Pág. 118
}

en términos de seguridad humana y su capacidad de desarrollar políticas de erradicación de procesos violentos $\mathrm{y}$ de prevención de cualquier ataque a los derechos $\mathrm{y}$ libertades fundamentales, se diera de manera brusca o como herramienta paliativa a una situación social de difícil contención ( sírvase comparar actualmente con algunas situaciones vividas a un nivel más reducido con la pandemia del COVID 19 en las grandes ciudades). Esta debe comenzar a corto plazo, dando peso específico a la ejecución local de las políticas de seguridad en contextos jurídicos coordinados por el Estado.

El titular ejecutivo del compromiso local, sería la importancia de los actores municipales en la planificación, ejecución y rendición de cuentas en el cumplimiento de los ODS. En este sentido el texto hace hincapié en algo básico si se quiere alcanzar en un futuro metas importantes, e insisto más aún en ciudades de carácter global o próximamente a serlo, al señalar que el objetivo es "hacer que las ciudades y los asentamientos humanos sean inclusivos, seguros, resilientes y sostenibles". Se indica que "la ODS 11 representa un gran paso hacia adelante en el reconocimiento del poder transformador de la urbanización para el desarrollo, y en el papel de los líderes de las ciudades a la hora de impulsar el cambio global desde la base". Es importante señalar la dicotomía que actualmente existe entre la realidad normativa española ${ }^{21}$ y la que se refleja en el programa de desarrollo de la Agenda 2030. Especialmente en la seguridad municipal (desde el plano de seguridad humana) se concreta en el desarrollo de políticas destinadas a paliar los efectos de la nueva urbanización, aumento de la población y polarización de ésta, cuando expresa que "Los GGLL españoles se sitúan en el ámbito territorial óptimo para la promoción del desarrollo sostenible a través de diferentes ámbitos: A través de la realización de actuaciones locales vinculadas con ODS, tales como los planes de movilidad urbana sostenible, la planificación de territorio, las estrategias de desarrollo sostenible locales, la promoción de políticas de igualdad y de seguridad ciudadana, los pactos locales por el empleo y la formación, o las políticas de cooperación". Así mismo y bajo la premisa del crucial papel de las entidades locales en la consecución de los ODS a nivel de Estado hace hincapié en aquellos relacionados con los servicios públicos esenciales y que su rol está a tres niveles "institucional incidencia política y alianzas." Pero ¿Los gobiernos locales pueden legislar? Obviamente en sentido genérico "sí”, a través de sus normas municipales, pero si nos referimos a legislar desde el plano jurídico del término es obvio que "no". No pueden hacer leyes en las que se atribuyan competencias para poder ejecutar y asegurar el cumplimiento de esta realidad tangible que es el aumento de las transferencias de poder del estado las grandes ciudades.

21 Quiero en este caso hacer mención a la L.O. de Fuerzas y Cuerpos de Seguridad. Es una norma que no ha sufrido modificación ninguna de calado, especialmente en lo que respeta al ámbito local desde su publicación y entrada en vigor en el año 86, desde hace casi 35 años, con las diferencias sociales y tecnológicas que actualmente existen en nuestra sociedad. 
Se debe dar más peso específico a las ciudades y que las autoridades municipales tengan un poder delegado del estado para ejecutar las políticas futuras. En este sentido van algunas de las medidas del Plan de Acción, que a mi juicio no se han visto reflejadas actualmente en actividad normativa ninguna y me temo que no se desarrollen de manera efectiva, especialmente las dirigidas al apoderamiento de la administración municipal. A título de ejemplo señalaré:

“a) Avanzar en el reconocimiento institucional de los poderes locales como nivel de gobierno con autonomía política plena que permita proceder a las reformas necesarias para mejorar el nivel de descentralización del país.

b) Fomentar planes de acción para la prevención de las violencias urbanas. Esto significa aumentar la colaboración y el intercambio de información entre las distintas administraciones, fomentando la supervisión y el cumplimiento de los tratados internacionales, las leyes y otros mecanismos para proteger los derechos humanos que ayuden a prevenir 1 violencia urbana".

c) Promocionar la dimensión local de las políticas palanca ${ }^{22}$ previstas en el plan, tanto en su diseño como en la ejecución y seguimiento".

Actualmente se están proyectando en la ciudad de Madrid la aplicabilidad de la Objetivos de desarrollo sostenible a tenor de los documentos de aplicación de nuestro país, y como hemos señalado anteriormente en el objetivo 11, que a mi juicio debe marcar un antes y un después en la concepción de las políticas y competentes de las administraciones locales, pero mucho más en las ciudades globales y grandes ciudades.

Madrid debe ser el líder de la apuesta de los municipios y administraciones locales en la consolidación de los ODS y sus planes programáticos deben estar alineados con las políticas internacionales en esta materia y especialmente desarrollando sus ideas entorno cinco pilares fundamentales que son las personas, el planeta, la prosperidad, la paz y las alianzas con terceros.

En este sentido el equipo de gobierno Municipal de Madrid ha impulsado de forma decidida el avance necesario destinado a la consecución de los ODS en Madrid. Y así en el pleno del Ayuntamiento de Madrid 25 de septiembre de 2019 por unanimidad aprobó lo siguiente:

- "La elaboración y aprobación por parte de la Junta de gobierno de una estrategia actualizada de localización, implementación y seguimiento de los objetivos de desarrollo sostenible de la agenda 2030 en el ámbito del municipio de Madrid, que tenga

22 Plan de Acción para la Implementación de la Agenda2030. Política palanca nos referimos a aquellos programas o políticas con capacidad de acelerar la implementación de los ODS, impulsar un desarrollo sostenible coherente y alcanzar un impacto más rápido y sostenido sobre aspectos claves para el progreso en el conjunto de la agenda 2030. Las políticas palanca han de entenderse como instrumentos transversales para romper silos o compartimentos sectoriales rígidos y atender a un desarrollo sostenible que conecte diferentes actores, sectores y política en una visión común integrada.” Pág. 130 en cuenta el borrador de estrategia y otros documentos relevantes ya desarrollados previamente.

- Que se arbitren los mecanismos que permitan tener en cuenta en dichas estrategias las anotaciones de los demás partidos políticos y otros agentes y actores relevantes de la ciudad tales como los representantes en el Foro Madrid solidaria.

- Que se desarrollen acciones necesarias para la divulgación y comunicación del contenido y alcance de la Agenda 2030 entre la ciudadanía madrileña.

Con carácter transversal y siendo fiel reflejo de la cooperación entre todos los actores (políticos, institucionales, sector privado), dentro de las políticas públicas de las administraciones, especialmente las administraciones más pegadas a la ciudadanía; se aprobó el día 7 de julio del año 2020 los denominados Pactos de la Villa, los primeros y únicos de este calado que fueron aprobados por unanimidad de todos los grupos políticos, ya que la pandemia que nos asola obliga a una respuesta eficaz para los residentes de la ciudad de Madrid.

Este planteamiento del Pleno Municipal (Pactos de la Villa) y la futura y próxima estrategia de aplicación de la Agenda 2030 hacen de Madrid un ejemplo determinante del nuevo concepto de "localización" de las políticas públicas, que en sentido general ya se establecía en el término "glocalidad" de la teoría del riesgo hace más de 50 años, especialmente en la idea de que problemas globales tienen su reflejo en el ámbito local.

\section{La aplicabilidad de los "ODS" en la seguridad pública madrileña}

De todos los ODS que podemos valorar en la política estratégica municipal de Madrid destaca el ODS 11, que ya ha sido mencionado en múltiples ocasiones en este texto, al ser el más relevante desde el plano de la seguridad humana en las ciudades globales. En el proyecto de cumplimiento del ODS 11 se ha dimensionado su cumplimiento a través de 109 actuaciones, 9 metas y 15 indicadores; pudiéndole calificar cuantitativamente del más importante.

La seguridad por tanto en Madrid tiene un carácter transversal para dar aplicabilidad y eficacia al cumplimiento de todos los objetivos. Una ciudad segura da confianza a la inversión y sostenibilidad a lo largo del tiempo. Desde las políticas de inversión y desarrollo de la seguridad de Madrid también se establecen objetivos operativos y metas de aplicabilidad para un eficaz cumplimiento de la agenda 2030.

Se puede destacar el análisis que desde la Dirección General de Policía Municipal se ha planteado para la inclusión de la seguridad en los objetivos de desarrollo sostenible, desde una perspectiva de seguridad humana global y de una seguridad subjetiva, donde el Cuerpo de Policía Municipal destaca como instrumento de políticas públicas que aglutine la puesta en marcha de metas concretas, que en algunos casos de visibilidad al proyecto de cumplimiento. 

ODS:

Proyectamos nuestra intervención en los siguientes

\section{a) ODS 1: Poner fin a la pobreza en todas sus formas en todo el mundo:}

Meta local: Reducción de las personas con sentimiento de soledad:

Las actuaciones que se desarrollan desde la esta Dirección General de Policía Municipal relacionado con la detección de este tipo de situaciones dentro de la Ciudad de Madrid, con ocasión de intervenciones requeridas en domicilios a través de las Unidades integrales de distrito, mediante las Oficinas de Atención ciudadana (OAC), y la Unidad de Atención y Protección a la Mujer, Mayor y Menor; así como la participación en el grupo motor de "Ciudades amigables con las personas mayores" se podrían sumar para la consecución de esta meta.

No olvidemos que en muchos casos este aislamiento, tare consigo patologías psiquiátricas que conducen a muertes provocadas o no provocadas, pero si asumidas. Es importante cuidar este sector vulnerable de la población que es propenso a una victimización indirecta de las circunstancias sociales (victima terciaria).

Es necesario reforzar los hábitos de las personas mayores ya que, en su caso, el aseguramiento de conductas trae consigo una reducción del sentimiento de inseguridad y por tanto podemos hacer que sean más libres con respecto a su comportamiento en sociedad.

\section{b) ODS 3: Salud y bienestar}

Meta 3.5 Agenda 2030 N.U.: Fortalecer la prevención y el tratamiento del abuso de sustancias adictivas, incluido el uso indebido de estupefacientes y el consumo nocivo de alcohol.

Con relación a esta meta, se podría crear una mesa local encaminada a la prevención en el ámbito de los menores de edad, la cual, estaría enmarcada en nuestro ámbito de actuación y concretamente dentro del programa "Participa en tu seguridad", que venimos desarrollando desde el Cuerpo de Policía Municipal. Continuando además con los protocolos de colaboración para la prevención de este tipo de consumo con Madrid Salud (Instituto de Adicciones) relacionados con la disposición adicional quinta de la ley de seguridad ciudadana de 2015: Suspensión de sanciones pecuniarias impuestas por infracciones en materia de consumo de drogas tóxicas, estupefacientes o sustancias psicotrópicas cometidas por menores de edad ${ }^{23}$.

23 Ley Orgánica 4/2015, de 30 de marzo, de Protección de la Seguridad Ciudadana. BOE 77 de 31/03/15 "Disposición adicional quinta. Suspensión de sanciones pecuniarias impuestas por infracciones en materia de consumo de drogas tóxicas, estupefacientes o sustancias psicotrópicas cometidas por menores de edad.

Las multas que se impongan a los menores de edad por la comisión de infracciones en materia de consumo o tenencia ilícitos de drogas tóxicas, estupefacientes o sustancias psicotrópicas podrán suspenderse siempre que, a solicitud de los infractores y sus representantes legales, aquéllos accedan a someterse a tratamiento o rehabilitación, si lo precisan, o a actividades de reeducación. En caso de que los infractores abandonen el tratamiento o rehabilitación o las actividades reeducativas, se procederá a ejecutar la sanción económica.

Reglamentariamente se regularán los términos y condiciones de la remisión parcial de sanciones prevista en esta disposición adicional."
Además, para la consecución de esta meta, participaremos con las actuaciones operativas que se establecen como compromiso a la ciudadanía en la Carta de Servicios del Cuerpo de Policía Municipal de Madrid.

Compromisos / tendencia: Informar a las familias sobre el contenido de la disposición y casos derivados a los Centros de Atención a las Adicciones en relación a las denuncias interpuestas por los componentes del Cuerpo de la Policía Municipal.

Establecer contactos y seguimientos con las familias de los menores denunciados y de aquellos casos comunicados por el Instituto de Adicciones al Servicio de agentes tutores de Policía Municipal.

Meta 3.6. Reducir un 50\% el número de muertes y lesiones graves en accidentes de tráfico.

Para la consecución de esta meta, actualmente, desde el Cuerpo de Policía Municipal, se está desarrollando un Plan de Seguridad Vial 2021 -2030, alineado con el Plan de Movilidad Sostenible Madrid 360, que recoge entre sus ejes estratégicos, "Avanzar hacia una Movilidad Segura", haciendo propio el objetivo estratégico de reducir en un $50 \%$ en el número de fallecidos y víctimas graves en accidentes de tráfico, en el próximo decenio. Para ello disponemos de los siguientes indicadores que se pueden referenciar a la consecución de esta meta:

- Indicadores asociados al Compromiso.

- Fallecidos por accidentes de tráfico.

- Heridos graves por accidentes de tráfico.

- Porcentaje de accidentes con víctimas en los que se llega al lugar del incidente en ocho o minutos o menos.

\section{c) ODS 4: Educación de calidad}

Meta 4.7 Agenda 2030 N.U.: De aquí a 2030, asegurar que todos los alumnos adquieran los conocimientos teóricos y prácticos necesarios para promover el desarrollo sostenible, entre otras cosas mediante la educación para el desarrollo sostenible y los estilos de vida sostenibles, los derechos humanos, la igualdad de género, la promoción de una cultura de paz y no violencia, la ciudadanía mundial y la valoración de la diversidad cultural y la contribución de la cultura al desarrollo sostenible.

Meta local:

1) Fomentar la formación de la población madrileña en ciudadanía global, igualdad de género, educación ambiental y consumo sostenible e igualando el peso relativo por género.

2) Fomentar la participación de los centros educativos públicos en programas y actividades municipales de educación ambiental.

3) Las metas relacionadas con la promoción de la cultura de paz y no violencia a través de acciones que ejecutaría el Cuerpo de Policía Municipal, se canalizarían mediante el Servicio de Agentes tutores, Unidad de Gestión de la Diversidad y aquellas relacionadas con materias medioambientales por la Unidad de Medioambiente. 
Para ello, las acciones de concienciación y prevención que se desarrollen, se incluirán dentro del programa "Participa en tu seguridad", que actualmente se está desarrollando desde el Cuerpo de Policía Municipal.

En este sentido el compromiso al igual que sucede con el resto de solicitudes recibidas en materia de formación e información, sería compromiso e información, sería impartir todas las charlas y conferencias preventivas en materia de civismo y respeto a la convivencia que sean solicitadas.

Indicadores asociados al compromiso: Reforzar y en su caso ampliar el número de charlas y conferencias impartidas.

\section{d) ODS 5: Igualdad de género}

Meta 5.2 Agenda N.U.: Eliminar todas las formas de violencia contra todas las mujeres y las niñas en los ámbitos público y privado, incluidas la trata y la explotación sexual y otros tipos de explotación.

Meta Local: Reducir de manera constante las víctimas de violencia de género.

Desde el Cuerpo de Policía Municipal se actúa de una forma proactiva desde la prevención, así como de forma reactiva en todas aquellas situaciones que lo requieran, como la participación y colaboración tanto en programas de otros Servicios del Ayuntamiento de Madrid, como en los programas propios en los que participarían la Unidad de Apoyo y Protección de la Mujer, Menor y Mayor (en adelante UAPMMM), Agentes Tutores, OAC. Aspectos que permitirán la consecución de esta meta.

Compromiso: Para apoyar y dar protección a las víctimas de violencia de género, Realizamos el seguimiento y protección de todas las víctimas asignadas, de acuerdo al Sistema VIOGEN.

Indicadores asociados al compromiso: Porcentaje de víctimas de violencia de género a las que se presta seguimiento y protección por parte de la Policía Municipal.

Igualmente se dispone de un Plan de Conciliación dentro del Cuerpo de Policía Municipal, que establece medidas internas de conciliación para situaciones relacionadas con la violencia contra las mujeres, que se suman a la consecución de la meta general y de forma proactiva.

\section{e) ODS 7: Energía asequible y no contaminante}

Meta 7.3 Agenda 2030 N.U.: Duplicar la tasa mundial de mejora de la eficiencia energética.

Meta local:

- Incrementar el uso de vehículos de cero emisiones.

- Lograr que la energía consumida en los edificios de viviendas que se construyan sea mayoritariamente renovable.

- Incrementar la producción energética basada en renovables dentro del objetivo general de autosuficiencia energética del Ayuntamiento de Madrid.

En el compromiso relacionado con el incremento de vehículos cero emisiones desde esta Dirección General, se participa con la incorporación paulatina de vehículos eco, cero etc., a la flota actual.

Igualmente, desde la esta Dirección General se priorizará que, en los nuevos proyectos de edificación de las nuevas sedes policiales, se implementen métodos de producción energética basada en energías renovables alineado con el objetivo general de autosuficiencia energética del Ayuntamiento de Madrid, aspecto que se suma a la consecución de las metas locales.

\section{f) ODS 11. Ciudades y comunidades sostenibles}

Meta 11.2 De aquí a 2030, proporcionar acceso a sistemas de transporte seguro, asequible, accesible y sostenible para todos y mejorar la seguridad vial, en particular mediante la ampliación del transporte público, prestando especial atención a las necesidades de las personas con vulnerabilidad, las mujeres, los niños, las personas con discapacidad y las personas de edad.

Meta local: Incrementar la utilización la bicicleta como medio de transporte potenciando el servicio público BiciMad.

Para la participación en la consecución de este objetivo, actualmente se están desarrollando acciones desde el Cuerpo de Policía Municipal, concretamente el programa "En Bici con la Policía Municipal", la participación en el proyecto "Stars" en los centros escolares que lo solicitan fomentando la participación de los mismos y la participación en el desarrollo de programas relacionados con el Camino Escolar Seguro al Cole, realizando planes integrales de seguridad en los entornos escolares, que fomentan la movilidad sostenible escolar y de creación de itinerarios peatonales escolares seguros.

Igualmente se realizan campañas y controles preventivos relacionados con la seguridad vial en el campo preventivo, como controles y campañas con datos que suman al compromiso de la Ciudad.

Compromiso: Realizar todas las actividades y conferencias solicitadas en esta materia.

Con objeto de favorecer la calidad de vida de la ciudadanía, y sumar a la consecución del objetivo 11 "lograr que las ciudades y los asentamientos humanos sean inclusivos, seguros, resilientes y sostenibles" se desarrollará un Plan Integral de Seguridad, Convivencia y Recuperación del Distrito.

En este sentido inicialmente se proyectará en los barrios identificados en los distritos municipales de Villa de Vallecas y Villaverde, de acuerdo a lo establecido en el Plan de Gobierno y Acuerdos de la Villa.

Compromiso: aprobación de un Plan Integral de Seguridad, Convivencia y Recuperación de barrios especialmente degradados.

Meta 11.4. Redoblar los esfuerzos para proteger y salvaguardar el patrimonio cultural y natural del mundo. Desde el Cuerpo de Policía Municipal, alineado con el Plan de Gobierno y Acuerdos de la Villa y con el objeto de potenciar la lucha contra las pintadas vandálicas en los edificios de Madrid, se pondrá en marcha un Plan grafitis o anti pintadas. 


\section{g) ODS 16. Paz, justicia e instituciones sólidas.}

Meta local: 16.1 Reducir significativamente todas las formas de violencia y las correspondientes tasas de mortalidad en todo el mundo. Desde la Dirección General de Policía Municipal se vela por mantener los niveles de seguridad y convivencia en la calle dando respuesta a la ciudadanía a través de su actuación, con objeto de mejorar los índices y percepción de la seguridad de ciudadanía. Para ello se establecen los siguientes indicadores, que participan en la consecución de la meta local.

Dentro del Plan de Gobierno existen además dos acciones que se desarrollarán relacionadas con este compromiso, como son el impulso de un nuevo Protocolo de Coordinación en materia de Policía Judicial junto con los Órganos Judiciales y otras Fuerzas y Cuerpos de Seguridad y la Aprobación de un Plan Integral de Lucha contra la "okupación ilegal".

Indicadores asociados

- Personas puestas a disposición judicial en relación con los delitos.

- Armas y objetos peligrosos relacionados con infracciones la Ley 4/2015 retirados de la vía pública.

- Porcentaje de incidentes urgentes en materia de seguridad en los que se llega al lugar del incidente en 8 minutos o menos desde que se produce la llamada.

- Índice de percepción de seguridad en la Ciudad.

Además, se desarrollarán acciones y programas mediante el servicio que prestan los Agentes Tutores, en los planes. "Participa en tu Seguridad", "Zonas libres de Acoso", "Programa Agente Mediador" y "Plan Integral de Seguridad Escolar”.

Compromisos: Mejorar la protección y la convivencia en el entorno escolar y zonas de ocio.

Indicadores asociados al al compromiso: Porcentaje de charlas y/o conferencias preventivas en materia de civismo y respeto a la convivencia impartidas por la Policía Municipal.

\section{Acciones}

- Incentivar la incorporación de centros escolares al compromiso de la erradicación del acoso escolar y cualquier tipo de violencia en las aulas a través del programa "Zonas Libres de Acoso"

- Atender las solicitudes de acciones formativas en gestión de conflictos y mediación entre iguales dirigidas a alumnos y alumnas menores de edad y jóvenes;

- Promocionar la implantación del servicio de mediación policial del Ayuntamiento de Madrid; así como los centros e instituciones adheridas al programa de mediación policial "Agente Mediador".

Indicadores asociados a la acción

- Número de acciones de divulgación y promoción de la mediación realizados como herramienta alternativa de gestión de conflictos y prevención.
- Porcentaje de solicitudes atendidas.

- Realizar el seguimiento de aquellos casos activos que puedan suponer algún tipo de riesgo para los menores en el ámbito de la protección y reforma, activando, si fuese necesario, a otras instituciones, durante el plazo mínimo de 2 meses.

- Porcentaje de casos de menores en los que se realiza seguimiento por la Policía Municipal.

- Mejorar la seguridad integral en los entornos y centros escolares.

- Vigilancias de entradas y salidas y recreos de centros escolares por la Policía Municipal.

- Mejorar la protección y la convivencia de las personas mayores de 65 años. Realizamos 60 estudios siguiendo la metodología del Plan PISE en los entornos de los centros frecuentados habitualmente por personas mayores. Impartimos todas las charlas y conferencias preventivas en materia de seguridad, civismo y respeto a la convivencia solicitadas.

- Estudios realizados a centros frecuentados por personas mayores por la Policía Municipal.

- Además, se realizará el seguimiento de todas las situaciones de riesgo, vulnerabilidad o maltrato judicializadas que afecten a las personas mayores de 65 años y sean comunicadas a la Policía Municipal, con respecto a las ya judicializadas y comunicadas a la Policía Municipal, haciendo un seguimiento del caso. La detección de estas situaciones puede hacerla la Policía Municipal o los servicios asistenciales municipales (SAMUR-Social, SAMUR-PC y Servicios Sociales), con los que se han establecido canales de comunicación a través de la Mesa de Vulnerabilidad.

- Porcentaje de situaciones de riesgo o vulnerabilidad que afecten a personas mayores de 65 años a las que se realiza seguimiento por la Policía Municipal.

- Situaciones de riesgo, vulnerabilidad o maltrato que afecten a personas mayores de 65 años detectadas por los servicios municipales (Policía y servicios asistenciales). Indicador informativo

- Porcentaje de aumento de las situaciones detectadas respecto a la judicializadas y comunicadas a la Policía Municipal.

Con relación a la Meta Local: Incrementar la administración electrónica en el Ayuntamiento de Madrid.

Se participa, con el compromiso / Tendencia: eliminación de trámites en papel en las comunicaciones internas y en la gestión administrativa: especialmente multas de tráfico, denuncias en general, actas y gestiones internas de forma electrónica y con la progresiva utilización de Tablet en la actividad policial.

Además, se implementa la gestión administrativa, en aquellas relaciones con la ciudadanía, como oposiciones, contestaciones mediante el sistema de sugerencias y reclamaciones, y aquellas contestaciones recogidas en el Reglamento de Participación Ciudadana del Ayuntamiento de Madrid.

Igualmente, y de forma progresiva se incrementa la gestión y administración electrónica, en todas aquellas 
relaciones de carácter interno, mediante herramientas informáticas, como CISEM, MOVIPOL, entre otras.

Meta Local: Contestar todas las reclamaciones y sugerencias presentadas

Compromiso: Contestamos el 75\% de las sugerencias, reclamaciones y felicitaciones como máximo en 30 días naturales y en ningún caso en un plazo superior a 2 meses.

\section{h) ODS 17. Alianzas para lograr los objetivos.}

Posible meta local: Conseguir una coherencia de políticas municipales con la Estrategia de Localización 2030 y lograrlo de forma participada.

Indicadores asociados:

- Planes de implementación realizados en las Direcciones Generales.

- Compromiso de Policía Municipal: plan de implementación de Agenda 2030, alineado en la Estrategia de la Dirección General de Policía Municipal.

Por otro lado, la Dirección General de la Policía Municipal del Ayuntamiento de Madrid, viene desarrollando proyectos institucionales internacionales con otros países de la Unión, en el ámbito de la aplicabilidad de la agenda 2030 en cuento a los ODS referidos a grandes ciudades y seguridad proactiva en correlación directa con la Nueva Agenda Urbana.

La Agenda Urbana para la UE representa un nuevo método de trabajo multinivel que promueve la cooperación entre las ciudades de los Estados miembros y otras entidades interesadas con el fin de mejorar la base de conocimientos sobre cuestiones urbanas. El principal objetivo es que autoridades locales, regionales, Estados Miembros e Instituciones Europeas trabajen conjuntamente para fortalecer la seguridad colectiva y la capacidad de resiliencia a través de intercambio de conocimientos y mejores prácticas, con el fin de crear una cultura positiva de la seguridad entre los ciudadanos europeos. Concepción de la seguridad como una responsabilidad compartida entre actores públicos y privados a diferentes niveles. Dentro de esta Agenda Urbana se incluyen diversas temáticas como transición energética, economía circular, movilidad urbana, etc., entre las que se encuentra seguridad en espacios públicos, conformándose el Partenariado de Seguridad en Espacios Públicos, del que la Dirección General de la Policía Municipal es parte.

El enfoque propuesto para obtener seguridad urbana requiere un equilibrio entre represión, prevención y cohesión social. La perspectiva para garantizar dicha seguridad urbana precisa por parte de las ciudades unos amplios procesos de participación. Se reconoce la necesidad de involucrar a la comunidad en el desarrollo de políticas de seguridad y su implementación.

En este plan de acción que se desarrolla por parte del Partenariado en Espacios Públicos, se establecen 3 áreas consideradas prioritarias, constituyéndose diferentes grupos de trabajo, los cuales se reparten de la siguiente manera:
1. Diseño y Planificación Urbana (coordinada por EFUS).

2. Tecnología/Seguridad para ciudades inteligentes y seguras (coordinada por Niza).

3. Dirección de la seguridad, compartiendo espacios públicos (coordinada por Madrid).

Consecuentemente al proyecto del Partenariado europeo del que somos parte, las acciones se describen operativamente para la consecución de los objetivos propuestos. En concreto la acción 6 del referido proyecto tiene como misión el desarrollo de una guía para el diseño arquitectónico y espacial (seguridad por diseño), que presenta evidentes interrelaciones con en el Proyecto "UrbSecurity (Urbact); De la planificación a ciudades más seguras", en el que participa igualmente la Dirección General de la Policía Municipal.

UrbSecurity es un proyecto europeo dentro del programa Urbact III (2014-2020), el cual se encuentra cofinanciado por el Fondo Europeo de Desarrollo Regional, los socios y los Estados miembros. Se encuadra dentro de "Agenda Urbana de la Unión Europea sobre Seguridad en Espacios Públicos", promulgada por la Comisión Europea, que persigue fomentar políticas urbanas novedosas de seguridad en Europa.

El objetivo es trabajar sobre el impacto de la planificación de las ciudades en la seguridad urbana, proporcionando a las mismas estrategias para aumentar la percepción de seguridad. Pretende mejorar la calidad de vida de los ciudadanos mediante la implementación de enfoques innovadores a la seguridad en el medio urbano.

La red UrbSecurity intenta analizar estrategias y conceptos del diseño y planificación urbana, los cuales pueden contribuir a prevenir la segregación y el comportamiento antisocial. De igual modo, el proyecto procura mejorar la percepción de los ciudadanos sobre la seguridad urbana. Es una red de 9 ciudades, incluida la Ciudad de Madrid, en el que Policía Municipal trabaja en la actualidad para la mejora de la seguridad de la Puerta del Sol.

\section{Conclusiones}

Primera: El concepto de sociedad del riesgo ya no es solo una teoría social, sino que es una evidencia que influye en la constitución de todos los aspectos humanos en la actualidad, tanto en lo conceptos políticos del riesgo y sus agendas, como en la estructura de las nuevas sociedades urbanas representadas por las ciudades globales.

Segunda: La economía representa el vértice de los riesgos globales e influye de manera derivada en otros factores de riesgo que tienen consecuencias importantes para la población mundial, especialmente en las ciudades globales, como son:

- Los riesgos medioambientales que ocupan un papel destacado en las nuevas políticas internacionales. Esto se debe principalmente al abaratamiento de los factores de producción industrial para con- 
seguir menores costes y mayor producción, considerando los inputs medioambientales factores económicamente caros y de poca rentabilidad.

- Los factores sociales, en referencia al desequilibrio social entre segmentos de población que trae consigo especialmente en las ciudades globales la constitución de guetos y marginalidad.

- Los movimientos geopolíticos preferentemente producidos por las políticas que satisfagan inmediatamente a la población más deprimida en contra de políticas técnicamente estructuradas a medio y largo plazo.

- Los riesgos tecnológicos son complementarios a los anteriores y tiene su origen en la rapidez de medios de producción con fines económicos.

Tercera: Debemos acudir a modelos de políticas públicas en seguridad global centradas en los riesgos globales, y solo a través de una legislación diferenciada en ese tipo de ciudades se pude dar una respuesta más eficaz. Para este fin contribuyen decididamente las políticas internacionales de empoderamiento y resiliencia urbana, como es el caso de la Nueva Agenda Urbana y los ODS.

Cuarta: El modelo de seguridad en cuanto a los nuevos retos por los riesgos globales, deben ser un modelo en el que el estado tenga las competencias legislativas, de coordinación e inteligencia y las entidades territoriales autonómicas y las ciudades globales (Madrid especialmente por sus características) deben de tener las competencias en ejecución y "alimentación" de información del sistema de seguridad nacional.

Quinta: El gobierno municipal de Madrid hace una apuesta decidida por la seguridad de la ciudad a través de políticas transversales desde las áreas de gobierno, aplicando el concepto de seguridad humana en todas sus actuaciones especialmente referidas a colectivos vulnerables.

\section{Documentos}

Madrid en el Mundo. Informe de Posicionamiento global 2020. Área de Economía, Innovación y Empleo. Ayuntamiento de Madrid. Diciembre 2019.

Convenio Marco Sobre Cambio Climático. Aprobación del Acuerdo de Paris. Naciones Unidas 2015.

Transformar nuestro mundo. La agenda 2030 para el Desarrollo Sostenible. Resolución aprobada por la Asamblea General de 2015

Nueva Agenda Urbana. Habitat III. Naciones Unidas. 2017.

Plan de Acción para la Implementación de la Agenda 2030. Hacia una Estrategia Española de Desarrollo Sostenible. 2018.

Fortalecimiento del movimiento Local-Global para localizar los Objetivos de Desarrollo Sostenible. Sevilla 2019.

World Economic Forum. Varios artículos.

Comunicación de la Comisión al parlamento Europeo, al Consejo Europeo, al Consejo, al Comité Económico y Social Europeo y al Comité de las Regiones sobre la Estrategia de la UE para la Unión de la Seguridad. Bruselas 24/07/2020. 